\title{
The effects of berberine on hyperhomocysteinemia and hyperlipidemia in rats fed with a long-term high-fat diet
}

\author{
Xin-xia Chang ${ }^{1}$, Hong-mei Yan', Qiong Xu', Ming-feng Xia ${ }^{1}$, Hua Bian ${ }^{1}$, Teng-fang Zhu ${ }^{2}$ and Xin Gao ${ }^{1 *}$
}

\begin{abstract}
Background: The study was undertaken to examine the effects of berberine (BBR) on serum homocysteine, lipids and the aortic lesion in Sprague-Dawley (SD) rats fed with a long-term high-fat diet (HFD).

Methods: Healthy male SD rats weighing 190-210 g received randomly standard diet or a high-fat diet for 24 weeks. After 8 weeks of feeding, rats fed with HFD were randomized to receive berberine $(200 \mathrm{mg} \cdot \mathrm{kg}-1 \cdot$ day-1) or vehicle by gavage for 16 weeks. After overnight fasting, all rats were sacrificed and total blood samples were also collected for determinant of fasting serum homocysteine (Hcy), total cholesterol (TC) and low density lipoprotein cholesterol (LDL-c) levels. The aorta was stained with hematoxylin and eosin (HE) and Sudan $\amalg$ to evaluate aortic lesion. The livers were dissected out and snap-frozen in liquid nitrogen for hepatic TC content and molecular analysis. 3-hydroxy-3-methyl-glutaryl-CoA reductase (HMGR), Lipoprotein receptors and apolipoproteins gene expression in the liver were determined by real-time PCR.
\end{abstract}

Results: Intragastrical administration with berberine for 16 weeks lowered serum Hcy in rats fed with a high-fat diet. In parallel, it also decreased body weight and improved serum TC and LDL-C. Berberine also tended to decrease hepatic cholesterol. Consistently, berberine also upregulated LDL receptor (LDLR) mRNA level and suppressed HMGR gene expression. Meanwhile, upon berberine-treated rats, there was a significant increase in apolipoprotein E (apoE) mRNA, but no change in apoAl and scavenger receptor (SR) mRNA in the liver. Further, no atherosclerotic lesions were developed in berberine-treated rats for 16 weeks.

Conclusion: Berberine can counteract HFD-elicited hyperhomocysteinemia and hyperlipidemia partially via upregulating LDLR and apoE mRNA levels and suppressing HMGR gene expression.

Keywords: Berberine, Hyperhomocysteinemia, Hyperlipidemia

\section{Background}

Homocysteine (Hcy) is a sulfur-containing amino acid formed during the metabolism of methionine. Hyperhomocysteinemia (HHcy) as a potent pro-inflammatory factor might accelerate the development of atherosclerosis [1]. Furthermore, the increased risk for vascular disease from elevated homocysteine is similar to that of other major cardiovascular risk factors. More importantly, it is independent of these factors [2-4]. Thus, reducing total Hcy levels can prevent the development of

\footnotetext{
*Correspondence: gao.xin@zs-hospital.sh.cn

'Department of Endocrinology and Metabolism, Zhongshan Hospital, Fudan University, Shanghai 200032, China

Full list of author information is available at the end of the article
}

arterosclerotic vascular disease. But, now it remains unclear whether berberine affects the level of serum Hcy.

Berberine (BBR), a natural alkaloid extracted from Coptis chinensis, is previously used for diarrhea treatment. In 1986, Chen et al. [5] at first reported that berberine can lower serum glucose levels, besides anti-inflammatory. Many studies [6-11] subsequently demonstrated that berberine has also beneficial effects in the improvement of lipid and glucose metabolism. Weight loss, lowering serum low density lipoprotein cholesterol (LDL-c) and glucose can prevent the development of arteriosclerosis. Thus, these studies suggested that berberine have the potential effect on preventing the development of atherosclerosis for these beneficial metabolic effects.

\section{Biomed Central}


However, it was recently reported that berberine promotes atherosclerosis development in apolipoprotein Edeficient $\left(\mathrm{apoE}^{-/-}\right)$mice, which could counter-balance the beneficial effect of lowering serum cholesterol [12]. $\mathrm{ApoE}^{-/-}$mouse is a well-established animal model for studying atherosclerosis [13-15], but, the pathogenesis in these mice is strikingly different from that of human atherosclerosis. Thus, the potential of berberine for affecting the level of serum homocysteine and atherosclerosis needs to be more carefully investigated. This study will observe the effects of berberine on atherogenic factors of atherosclerosis (e.g. serum Hcy and lipids) and the aortic lesion in wild rats fed with a long-term high-fat diet.

\section{Results}

Berberine lowered serum hcy and improved typical risk factors related to atherosclerosis in rats fed with a highfat diet (HFD)

The body weight of SD rats was gradually increased with a high-fat diet for 24 weeks. Body weights of HFD-fed rats were significantly reduced in BBR -treated group for 16 weeks (Figure 1A) as compared with control group with no dramatic changes in food intake (Figure 1B).

Intragastrical administration with BBR for 16 weeks, serum homocysteine was significantly decreased by about $60 \%$ in contrast to the vehicle-treated rats fed with the same high-fat diet $(p<0.001$, Figure 2A). BBR treatment significantly lowered the levels of serum TC $(p<$ 0.05 , Figure $2 \mathrm{~B})$ and LDL-C $(p<0.05$, Figure $2 \mathrm{C})$ as compared with the placebo group in SD rats fed with a high-fat diet. These data suggested that BBR exert antagonizing effects on HFD-induced dyslipidemia and hyperhomocysteinemia in SD rats.

\section{BBR decreased hepatic cholesterol in rats fed with HFD}

The effects of berberine on total cholesterol level were measured in rat liver. Rats fed with HFD alone showed a significant $(p<0.001)$ increase in hepatic cholesterol content, when compared with the normal controls (Figure 3). Treatment of high-fat diet-fed rats with berberine (200 mg/kg/d) tended to reduce hepatic cholesterol level, although this difference failed to reach statistical significance when compared to the high-fat diet control.

\section{Berberine reverses HFD-elicited abnormal expression of} some key genes related to cholesterol metabolism in the liver

The effects of berberine on 3-hydroxy-3-methyl-glutaryl-CoA reductase (HMGR), lipoprotein receptors and apolipoproteins gene expression in the liver of high-fat diet-fed rats were investigated. The relative mRNA levels of low-density lipoprotein receptor (LDLR) $(p<0.05$, Figure $4 \mathrm{~A})$ and apoE $(p<0.05$, Figure $4 \mathrm{~B})$ was significantly down-regulated in the livers of HFD-fed rats

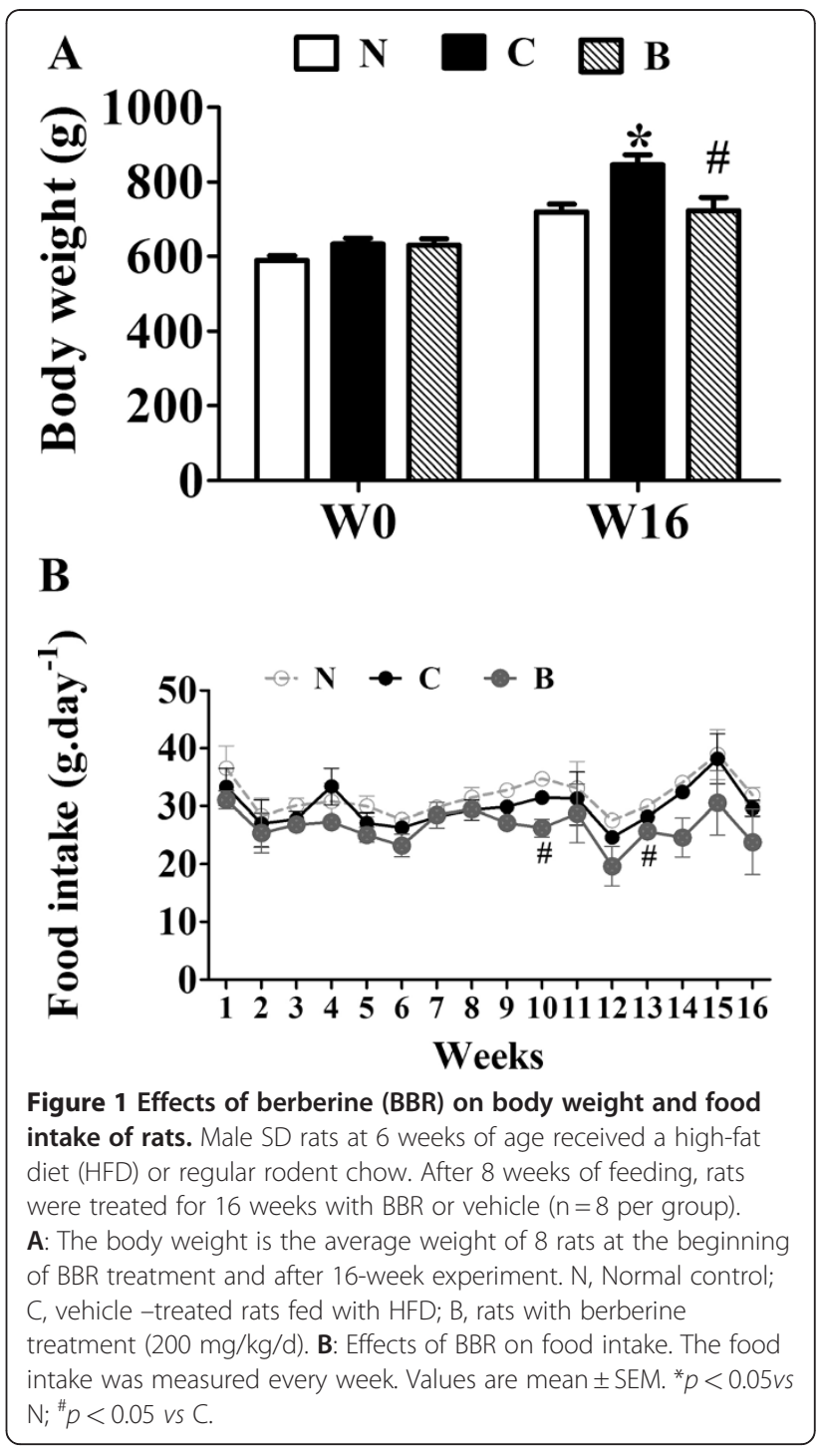

relative to the ND control group. BBR treatment significantly reversed the downregulating effects of HFD on the expression of LDLR and apoE $(p<0.05)$. On the other hand, the level of HMGR mRNA was increased in rats fed with long-term high-fat diet, which was markedly lowered by BBR treatment ( $p<0.05$, Figure 4A). We also found that apoB and apoAI mRNAs were suppressed by HFD feeding $(p<0.05$, Figure $4 \mathrm{~B})$, but these were not affected by BBR treatment. Despite these changes, BBR and high-fat-diet feeding did not influence the expression of scavenger receptor (SR).

Effect of BBR treatment for 16 weeks on aortic lesions in rats fed with a high-fat diet

Histological analysis by HE staining of the aorta showed that the tunica intima of aorta remained smooth and intact in rats with a 24- weeks high-fat diet after treatment with berberine for 16 weeks (Figure 5A-D). The results 


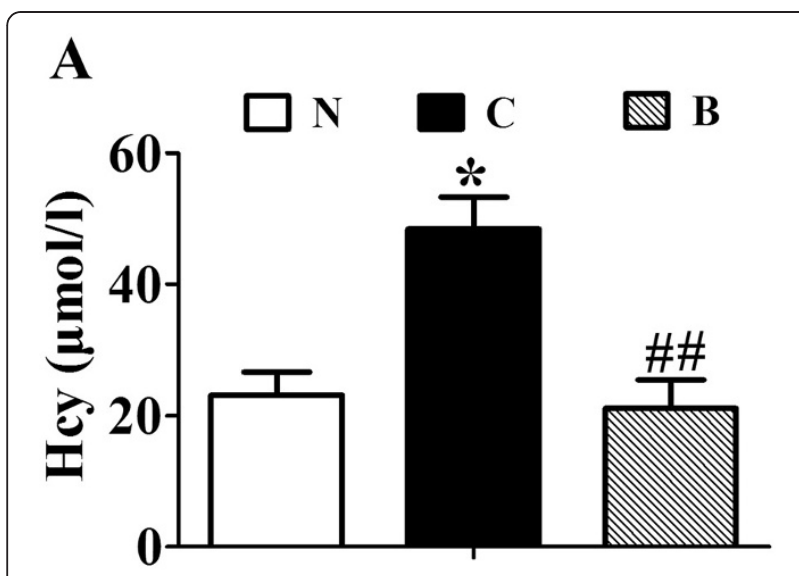

B
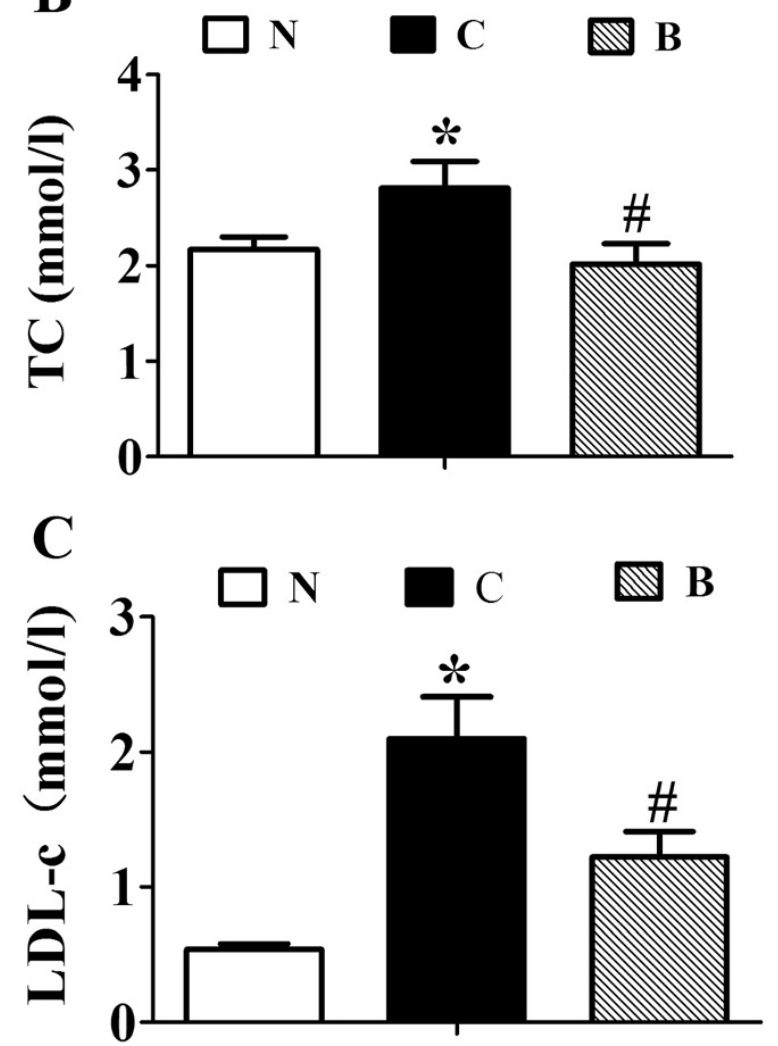

Figure 2 Effects of berberine (BBR) on serum Hcy and lipid profile. Serum Hcy $(\mathbf{A}), T C(\mathbf{B})$ and LDL-C $(\mathbf{C})$ are the average of each group $(n=8)$. N, Normal control; $C$, vehicle -treated rats fed with HFD; $B$, rats with berberine treatment $(200 \mathrm{mg} / \mathrm{kg} / \mathrm{d})$. Values are mean \pm SEM. ${ }^{*} p<0.05$ vs $N$; ${ }^{*} p<0.05$ vs $C$.

of aorta stained with Sudan III showed that no lipid inclusions and foam cell accumulation were seen in the aortic endothelium of these rats (Figure 5E-H). Furthermore, in other rats fed with a high-fat diet without administration of berberine, there were also no deposits of monocyte-derived macrophages and abnormal fatty

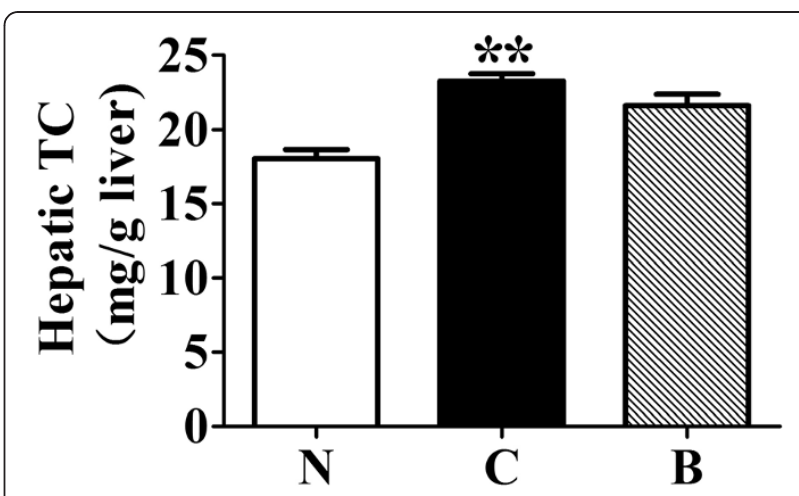

Figure 3 Effects of berberine (BBR) on hepatic total cholesterol. N, Normal control; C, vehicle -treated rats fed with HFD; B, rats with berberine treatment $(200 \mathrm{mg} / \mathrm{kg} / \mathrm{d})$. Values are mean \pm SEM. Significance was assessed by one-way ANOVA followed by Tukey's Multiple Comparison test. Data are mean \pm SEM. ${ }^{*} p<0.05,{ }^{* *} p<0.01$ vs N.

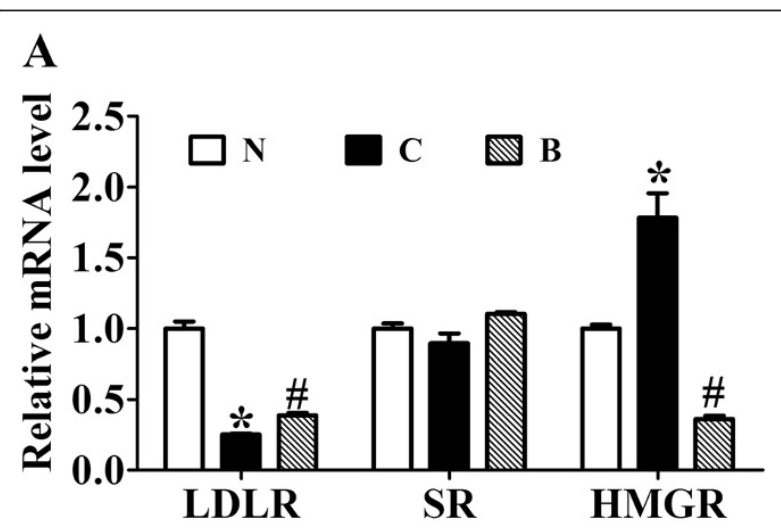

B

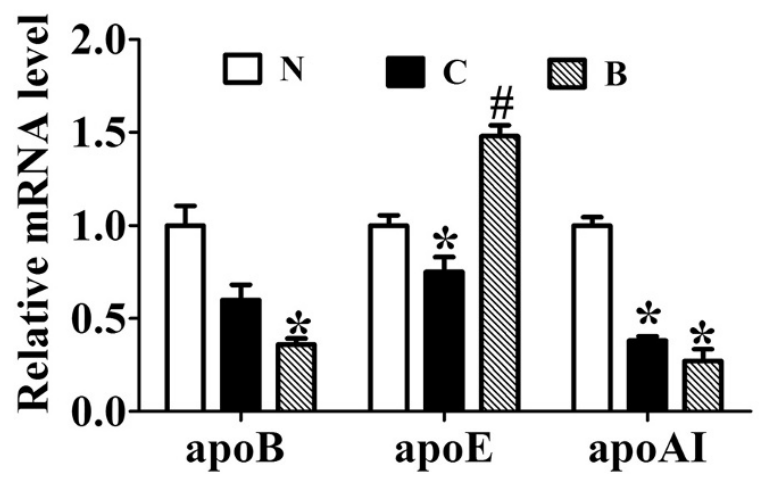

Figure 4 The effects of berberine on 3-hydroxy-3-methylglutaryl-CoA reductase (HMGR), Lipoprotein receptors and apolipoproteins gene expression. Real-time quantitative $P C R$ analysis of LDLR, HMGR and SR (A) and apolipoproteins (B) in the livers of SD rats administrated with berberine or vehicle $(n=8$ per group). Relative mRNA amounts of each gene were normalized to that of beta-actin. Values are mean \pm SEM. ${ }^{*} p<0.05$ vs N; ${ }^{\#} p<0.05$ vs $C$. 


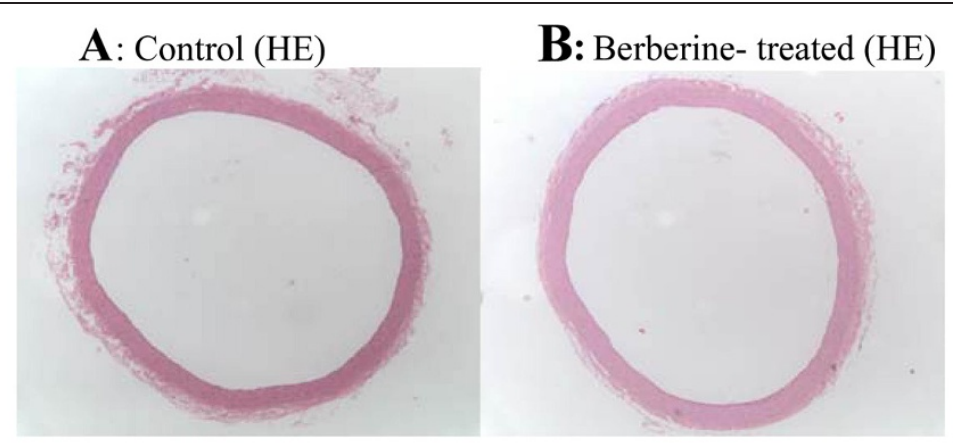

(Photographs are at $\times 50$ magnification)

C: Control (HE)

D : Berberine- treated (HE)

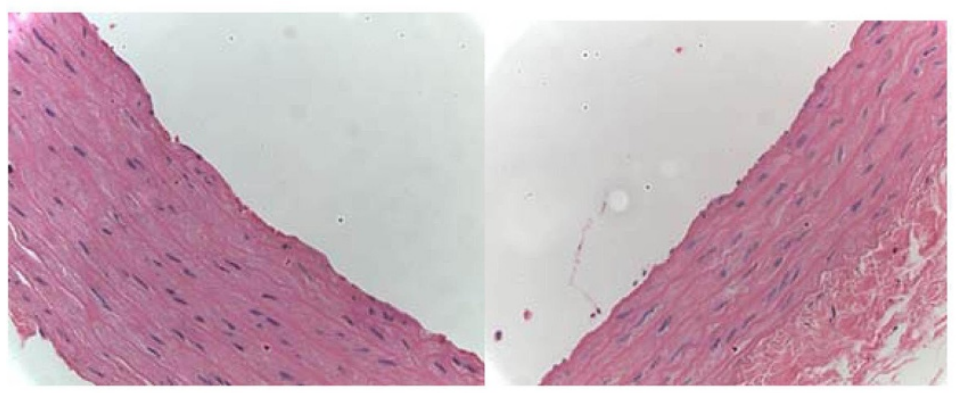

(Photographs are at $\times 400$ magnification)
E: Control (Sudan ш)
F: Berberine- treated (Sudan w)

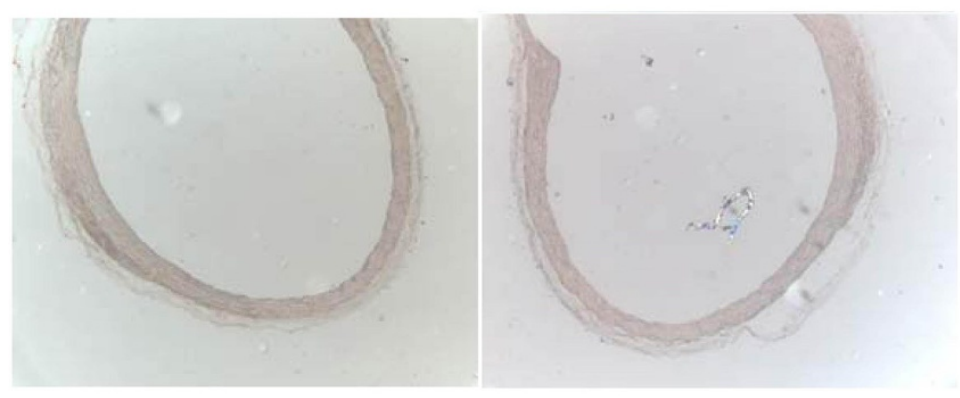

(Photographs are at $\times 50$ magnification)
G: Control (Sudan ш)
H: Berberine- treated (Sudan ш)

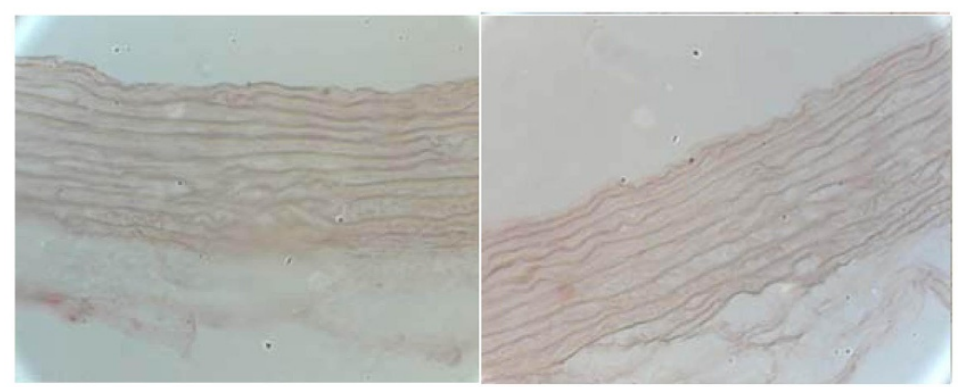

(Photographs are at $\times 400$ magnification)

Figure $\mathbf{5}$ (See legend on next page.) 
droplets in aortic tunica intima. These results suggested that no atherosclerotic lesions were developed in berberine-treated for 16 weeks and control rats fed with a long-term high-fat diet.

\section{Discussion}

In this study, we have shown that berberine could decrease serum homocysteine level and many known risk factors related to atherosclerosis, such as body weight, serum cholesterol, and LDL-c in SD rats fed with a high-fat diet. Consistently berberine could upregulate LDLR and apoE, and downregulate HMGR gene expression in the liver. Importantly, our morphological results demonstrated that berberine do not increase the development of atherosclerosis in SD rats fed with a longterm high-fat diet.

Recent studies demonstrate that hyperhomocysteinemia (HHcy) as a potent pro-inflammatory factor increases the risk for the development of atherosclerosis, which is independent of these typical cardiovascular risk factors [1-4]. The results of a meta-analysis [16] including 27 studies relating homocysteine to arteriosclerotic vascular disease and 11 studies of folic acid effects on serum homocysteine levels population-based case-control studies showed that a 5-mu mol/L total Hcy (tHcy) increment causes a 1.6-fold and a 1.8-fold increase in risk for coronary artery disease (CAD) for men and women, respectively. Further, a total of $10 \%$ of the population's CAD risk appears attributable to tHcy. The OR for cerebrovascular disease $(5-\mathrm{mu} \mathrm{mol} / \mathrm{L}$ tHcy increment) is 1.5 (95\% CI, 1.3 to 1.9 ) and Peripheral arterial disease also showed a strong association. The present study showed that rats fed with HFD alone $(n=8)$ showed a significant increase in serum Hcy at the end of 16 weeks' treatment, when compared with the normal controls fed a standard diet alone. High-fat diet-fed rats treated with berberine exhibited a significant reduction in serum Hcy by 1.3-fold compared with the high-fat diet treated rats alone. BBR can decrease homocysteine, which indicated that berberine have a beneficial effect in preventing atherosclerosis.

Dyslipidemia is the most important modifiable risk factor of atherosclerosis. The present study demonstrated that administration of berberine along with the high-fat diet effectively reduced serum TC and LDL-c in concordance with other reports [6-11]. Plasma cholesterol level is determined by the net balance between the input of cholesterol into plasma (biosynthesis) and removal rate from plasma $[17,18]$. The former is mainly dependent on the availability of the rate-limiting enzyme, HMG-CoA reductase (HMGR). High level of hepatic $L D L R$ mRNA is associated with improved clearance of plasma LDL-c [17]. Apolipoproteins are carrier proteins that bind lipids to form lipoprotein particles and also serve as enzyme cofactors and lipid transfer carriers that regulate the metabolism of lipoprotein. Thus, the removal rate from plasma in part is related to the level of LDLR and apolipoproteins. We measured mRNA of lipoprotein receptors, apolipoproteins and HMGR in the liver of rats after chronic treatment with berberine. We observed that administration of berbeine significantly upregulated LDLR and apoE gene expression in the liver of rats fed with a high-fat diet. On the contrary, elevated gene expression of hepatic HMGR in long-term HFDfed rats was down-regulated upon treatment of berberine. Apolipoprotein E (apoE) is synthesized by the liver that mediates the transport and uptake of cholesterol and lipid by way of its high affinity interaction with different cellular receptors, including the low-density lipoprotein (LDL) receptor[19]. One study showed that apoE deficiency causes high serum cholesterol and triglyceride levels and leads to premature artherosclerosis [20]. The previous study indicated that BBR reduces serum cholesterol, LDL-cholesterol via elevating hepatic $L D L R$ gene expression through a post-transcriptional mechanism that stabilizes its mRNA [7]. Thus, BBR might increase more clearance of serum LDL-c through its action on LDLR and apoE and reduce cholesterol biosynthesis by suppressing HMGR gene expression.

ApoB is the primary apolipoproteins of chylomicrons and low-density lipoproteins. While it is unclear exactly what functional role apoB plays in LDL, it is the primary apolipoprotein component and is absolutely required for its formation. Lipids are normally exported from the liver in very-low-density lipoproteins (VLDLs), which are complex lipoprotein particles that involve the fusion of a newly synthesized apolipoprotein B-100 (apoB-100) molecule with a triglyceride droplet through the action of microsomal triglyceride transfer protein (MTTP) [21]. A reduction in MTTP function and apoB synthesis and secretion may impair hepatic lipid export and favour triglyceride accumulation in the liver [22,23]. In our previous study [11], long-term high-fat-diet induced significant fat accumulation in rat liver via inhibiting the 
assembly and secretion of VLDL due to reduction in MTTP function. In this study, apoB gene expression was down-regulated in the liver of rats fed with long-term high-fat-diet compared with normal control rats, but berberine did not affect it. Thus, these suggested that long-term high-fat diet could simultaneously reduce apoB synthesis to favor fat accumulation in the liver, which needs further investigation.

Meanwhile, apoAI mRNA level was decreased in the liver of rats fed with long-term high-fat-diet compared with normal control rats, but berberine also did not affect it.

Atherosclerosis is a systemic inflammatory disease which is associated with several genetic and environmental risk factors. Epidemiologic, clinical and experimental studies show that Long-term intake of highcalories food leads to metabolic derangements characterized by hyperlipidemia and hyperglycemia, which induced the development of atherosclerosis. Thus, SD rats fed with a high-fat diet is a well-established animal model of reproducing the risk factors of premature atherosclerosis development in human [24-27].

Atherosclerosis/angiostasis partially stems from the injury or phenotypic alteration of endothelial cells (ECs), the cells in the frontline against vascular disturbances [28]. The histological examination of HHcy rats revealed an increased recruitment of monocytes to aortic endothelium accompanied by elevated immunostaining for monocyte chemoattractant protein-1, vascular cell adhesion molecule-1 and E-selectin [29]. The present study showed that no abnormal fatty deposits in the intima of arteries were observed and the intima of aorta remained intact in rats fed with a long-term high-fat diet after administration of berberine by gavage for 16 weeks. So berberine could prevent the aortic lesion, which may partially be through above-mentioned mechanism.

Although morphological results also showed no atherosclerotic lesions developed in control group, berberine can improve serum homocysteine and several typical risk factors associated with atherosclerosis, and there is not atherosclerotic lesions observed in berberine-treated group. These results at least suggest that berberine does not promote atherosclerosis development in SD rats fed with long-term high-fat diet.

In contrast to the present results, a recent study showed that berberine can induce foam cell formation and promotes atherosclerosis development in apolipoprotein Edeficient (apoE-/-) mice, which could counter-balance the beneficial effect of lowering serum cholesterol [12]. And the apoE-/- mice are gene-deficient animal model, in which targeted deletion of apoE gene leads to severe hypercholesterolemia and spontaneous. The apoE-/model has been used widely due to the rapid development of atherosclerosis, despite considerable limitations. A major fault is that plasma cholesterol level is about $8 \mathrm{mmol} / \mathrm{L}$ on chow diet, compared with $2 \mathrm{mmol} / \mathrm{L}$ for the parent $\mathrm{C} 57 \mathrm{Bl} / 6$ mouse, and can increase $>70 \mathrm{mmol} / \mathrm{L}$ on a high-fat, high-cholesterol (HFC) diet [30]. Another main shortcoming is that most plasma cholesterol is confined to VLDL and not to LDL articles as in humans. Therefore, the different results of these studies are mainly due to the animal model. Our present study at least demonstrated that berberine did not increase the risk of atherosclerosis in SD rats fed with a long-term high-fat diet, although these rats are not the typical model of atherosclerosis.

In summary, the present study has demonstrated that berberine can decrease serum homocysteine and several traditional risk factors associated with atherosclerosis via regulating LDLR, apoE and HMGR gene expression in rats fed with high-fat diet. Our findings illustrate that berberine may represents a promising agent for the pristine prevention of atherosclerosis.

\section{Methods}

Berberine, serum triglyceride determination Kit, and LDL-c Kit were purchased from Sigma-Aldrich, St. Louis, MO.

\section{Animal experiment}

The animal component of this study has been described in detail in our recently published data [11]. Briefly, healthy male Sprague -Dawley (SD) rats [Grade SPF, certificate number of the breeder: $\operatorname{SCXK}(\mathrm{Hu})$ 2007-0005] weighing 190-210 g from the Animal Development Center (Chinese Academy of Sciences, Shanghai, China) were given free access to food and water and were maintained on a 12/12-hour light/dark cycle. Rats received either a regular rodent chow (normal diet: $62.3 \%$ carbohydrate/ $12.5 \%$ fat $/ 24.3 \%$ protein calories) or a high-fat diet (32.6\% carbohydrate $/ 51.0 \%$ fat $/ 16.4 \%$ protein calories) for 24 weeks. After 8 weeks of feeding, rats on the HFD received randomly intragastric injection of either berberine $\left(200 \mathrm{mg} \cdot \mathrm{kg}^{-1} \cdot \mathrm{day}^{-1}\right)$ or vehicle for 16 weeks. Rats fed the normal diet received the equal volume of vehicle ( $0.5 \%$ methylcellulose, $\mathrm{N}$ group) as a control group. After an overnight fasting period, blood samples were also collected for measurement of serum Hcy, TC and LDL-C levels using commercially available kits. The liver tissues were stored in liquid nitrogen for biochemical and molecular analysis.

All experimental procedures involving the use of animals were conducted in conformity with PHS policy and were approved by the Animal Use and Care Committee of Fudan University.

\section{Measurement of serum hcy by ELISA}

Serum homocysteine ELISA kit was from Maibiotechnology, China. Add 100 $\mu$ l each of dilutions of standard, 
blank and samples into the appropriate wells. Mix well and incubate for 2 hours at $37^{\circ} \mathrm{C}$. Remove the liquid of each well, and wash with 400 of $1 \mathrm{X}$ Wash Solution to each well. Then add $100 \mu \mathrm{l}$ of biotinylated antibody working solution to each well. After incubate for 1 hour, washing the wells. Add $100 \mu$ l of enzyme conjugate working solution to each well. Incubate for 30 minutes at $37^{\circ} \mathrm{C}$ after covering it with the Plate sealer. Aspirate the solution and wash with $400 \mu \mathrm{l}$ of $1 \mathrm{X}$ Wash Solution to each well. Then add $100 \mu$ of TMB solution working solution to each well. Incubate for 15 minutes at $37^{\circ} \mathrm{C}$ after covering it with the Plate sealer. Protect from light. Add $50 \mu \mathrm{l}$ of Stop Solution to each well. Then, run the microplate reader and conduct measurement at $450 \mathrm{~nm}$ immediately.

\section{Measurement of serum TC}

Amplex red cholesterol assay kit (A12216) was obtained from invitrogen detection technologies. Prepare a cholesterol standard curve. Dilute serum in 1XReaction Buffer. Pipet $50 \mu$ l of the diluted samples and controls into separate wells of a microplate. Begin the reactions by adding $50 \mu \mathrm{l}$ of the Amplex Red reagent/HRP/cholesterol oxidase/cholesterol esterase working solution to each microplate well containing the samples and controls. Incubate the reactions for 30 minutes at $37^{\circ} \mathrm{C}$, protected from light. Measure the fluorescence in a fluorescence microplate reader using excitation in the range of 530$560 \mathrm{~nm}$ and emission detection at $\sim 590 \mathrm{~nm}$. According to the standard curve, get the cholesterol level of each sample.

\section{Measurement of serum LDL-c}

Prepare working solution containing cholesterol oxidase/ cholesterol esterase/catalase/peroxidase. Pipet $5 \mu$ l of the diluted samples and controls into separate wells of a microplate. Then add $500 \mu$ l of working solution to each microplate well. Mix well and incubate the reactions in the $37^{\circ} \mathrm{C}$ water bath for 10 minutes. Measure the absorbance value at $546 \mathrm{~nm}$.

\section{Hepatic total cholesterol level}

Hepatic lipids were extracted according to the method of Folch et al. [31]. Briefly, lipid was extracted from frozen liver tissues $(30 \mathrm{mg})$ by homogenization in $1 \mathrm{ml}$ of 2:1 chloroform: methanol, followed by shaking at room temperature for overnight and centrifugation at $3000 \mathrm{rpm}$ for $10 \mathrm{~min}$. Aliquots $(400 \mathrm{l})$ of the organicextract lipid suspension were analyzed for hepatic total cholesterol concentration using commercial diagnostic kits. Hepatic TC content was defined as mg of cholesterol per gram of the liver.
Table 1 Primer sequences for real-time quantitative PCR

\begin{tabular}{|c|c|c|}
\hline Gene & Forward primer $\left(5^{\prime}-3^{\prime}\right)$ & Reverse primer $\left(5^{\prime}-3^{\prime}\right)$ \\
\hline LDLR & CCAGTGCGGCGTAGGATT & GGGACTCATCGGAGCCAT \\
\hline HMGR & AGAATATAGCGCGTGGGATG & GACATACAGCCAAAGCAGCA \\
\hline apoB & TAAATGGAGCACTTITCAAG & GGAACAGCAGCAGTAGCG \\
\hline apoAl & GCCACTGTGTATGTGGATGC & AACCCAGAGTGTCCCAGTTG \\
\hline apoE & AGGAGCAGACCCAGCAGATA & GGAGTTGGTAGCCACAGAGG \\
\hline SR & GCCACTGGTCCTTGTTTGT & TGGGAGCGCTGACTITTACT \\
\hline beta-actin & GATTACTGCCCTGGCTCCTA & TCATCGTACTCCTGCTTGCT \\
\hline
\end{tabular}

\section{Histological analysis}

After the rats ( $\mathrm{n}=8$ pre group) were sacrificed, the aortas were removed from the region of the proximal aorta and fixed in phosphate-buffered 10\% formalin, which was subsequently stained with hematoxylin and eosin (HE). The aorta was then divided into 2 sections, one of which was embedded in paraffin blocks and the other in O.C.T. compound. A section from each paraffin block was stained with HE to examine the pathologic structures of the aorta and serial cryosections (five consecutive slices each sample) were stained with Sudan Ш to evaluate aortic lesion. These pathological slices were observed by the experienced pathologists in blinded state.

\section{Real-time quantitative PCR (qPCR) analysis}

Total RNA was isolated from liver tissues using Trizol reagent (Invitrogen, Carlsbad, CA, USA). cDNA was synthesized by reverse transcription using ReverTra Ace (Toyobo, Osaka, Japan). The SYBR Green PCR Master Mix (Toyobo, Osaka, Japan) was used for qPCR with a sequence detection system (ABI PRISM7900, Applied Biosystems, Foster City, CA, USA). Eight $\mu \mathrm{L}$ reaction mixture contained $1 \mu \mathrm{L}$ of $\mathrm{cDNA}$ and $125 \mathrm{nmol} / \mathrm{L}$ of primers. The specific primers of PCR amplification are described in Table 1 . The same reaction was performed in triplicate with The relative gene expression was calculated using the $2^{-\Delta \Delta \mathrm{Ct}}$ as described previously [32].

\section{Statistical analysis}

All data were presented as mean \pm SEM. Significance was assessed by Sudent's $t$-test. All $p$ values were twotailed and $p$ values of less than 0.05 were considered to be statistically significant.

\section{Abbreviations}

BBR: Berberine; SD: Sprague -Dawley; HFD: High-fat diet; Hcy: Homocyteine; TC: Total cholesterol; LDL-C: Low density lipoprotein cholesterol; HE: Hematoxylin and eosin; HMGR: 3-hydroxy-3-methyl-glutaryl-CoA reductase; LDLR: LDL receptor; apoE: Apolipoprotein E; SR: Scavenger receptor; HHcy: Hyperhomocysteine; apoE-/-: Apolipoprotein E-deficient; tHcy: Total Hcy; CAD: Coronary artery disease; VLDL: Very low density lipoprotein; MTTP: Microsomal triglyceride transfer protein; ECs: Endothelial cells; HFC: High-fat, high-cholesterol; qPCR: Real-time quantitative PCR. 


\section{Competing interests}

The authors declare that they have no competing interests.

\section{Authors' contributions}

$X G$ designed the research and revised this manuscript; $X C$ and $H Y$ carried out animal experiment and wrote the paper; QX performed parts of western immunoblot analysis and helped to draft the manuscript; $H B$ and $M X$ participated in parts of data analysis; TZ performed Histological analysis. All authors read and approved the final manuscript.

\section{Acknowledgements}

The authors thank Yong LIU (The Institute for Nutritional Sciences, Chinese Academy of Sciences, China) and Qi-qun TANG, Xi LI (The Institute of Biomedical Sciences, Fudan University, China) for their help. The authors are most grateful to Jian-zong GU and Lei LIANG (Shanghai Experimental Animal Center, China Academy of Sciences, China) for their help with animal studies. This study was supported by grants from the National Basic Research Program of China (Grant 2011 CB504004 and 2012CB524906 to Xin GAO), 985III-YFX 0302 to Xin GAO and National Natural Science Foundation of China (Grant 81100602 to Xin-xia CHANG).

\section{Author details}

'Department of Endocrinology and Metabolism, Zhongshan Hospital, Fudan University, Shanghai 200032, China. ²Department of Pathology, Shanghai Medical College, Fudan University, Shanghai 200032, China.

Received: 12 February 2012 Accepted: 25 June 2012 Published: 4 July 2012

\section{References}

1. McCully KS: Hyperhomocysteinemia and arteriosclerosis: historical perspectives. Clin Chem Lab Med 2005, 43:980-986.

2. Graham IM, Daly LE, Refsum HM, Robinson K, Brattstrom LE, Ueland PM, et al: Plasma homocysteine as a risk factor for vascular disease. The European Concerted Action Project. JAMA 1997, 277:1775-1781.

3. McCully KS: Homocysteine and vascular disease. Nat Med 1996, 2:386-389.

4. Guthikonda S, Haynes WG: Homocysteine as a novel risk factor for atherosclerosis. Curr Opin Cardiol 1999, 14:283-291.

5. Chen QM, Xie MZ: Studies on the hypoglycemic effect of Coptis chinensis and berberine. Yao Xue Xue Bao 1986, 21:401-406

6. Kim WS, Lee YS, Cha SH, Jeong HW, Choe SS, Lee MR, et al: Berberine improves lipid dysregulation in obesity by controlling central and peripheral AMPK activity. Am J Physiol 2009, 296:E812-E819.

7. Kong W, Wei J, Abidi $P$, Lin M, Inaba S, Li C, et al: Berberine is a novel cholesterol-lowering drug working through a unique mechanism distinct from statins. Nature medicine 2004, 10:1344-1351.

8. Yin J, Gao ZG, Liu D, Liu ZJ, Ye JP: Berberine improves glucose metabolism through induction of glycolysis. Am J Physiol Endocrinol Metab 2008, 294:E148-E156.

9. Kong WJ, Zhang H, Song DQ, Xue R, Zhao W, Wei J, et al: Berberine reduces insulin resistance through protein kinase $\mathrm{C}$-dependent up-regulation of insulin receptor expression. Metab Clin Exp 2009, 58:109-119.

10. Lee YS, Kim WS, Kim KH, Yoon MJ, Cho HJ, Shen Y, et al: Berberine, a natural plant product, activates AMP-activated protein kinase with beneficial metabolic effects in diabetic and insulin-resistant states. Diabetes 2006, 55:2256-2264.

11. Chang $X$, Yan H, Fei J, Jiang M, Zhu H, Lu D, et al: Berberine reduces methylation of the MTTP promoter and alleviates fatty liver induced by a high-fat diet in rats. Jounal of Lipid Research 2010, 51:2504-2515.

12. Li K, Yao WQ, Zheng XD, Liao K: Berberine promotes the development of atherosclerosis and foam cell formation by inducing scavenger receptor A expression in macrophage. Cell Research 2009, 19:1006-1017.

13. Zhang SH, Reddick RL, Piedrahita JA, Maeda N: Spontaneous Hypercholesterolemia and Arterial Lesions in Mice Lacking Apolipoprotein-E. Science 1992, 258:468-471.

14. Plump AS, Smith JD, Hayek T, Aaltosetala K, Walsh A, Verstuyft JG, et al: Severe Hypercholesterolemia and Atherosclerosis in Apolipoprotein-EDeficient Mice Created by Homologous Recombination in Es Cells. Cell 1992, 71:343-353.
15. Reddick RL, Zhang SH, Maeda N: Atherosclerosis in Mice Lacking Apo-E Evaluation of Lesional Development and Progression. Arterioscler Thromb 1994, 14:141-147.

16. Boushey CJ, Beresford SA, Omenn GS, Motulsky AG: A quantitative assessment of plasma homocysteine as a risk factor for vascular disease. Probable benefits of increasing folic acid intakes. JAMA 1995, 274:1049-1057.

17. Brown MS, Goldstein JL: A receptor-mediated pathway for cholesterol homeostasis. Science 1986, 232:34-47.

18. Russell DW: Cholesterol biosynthesis and metabolism. Cardiovasc Drugs Ther 1992, 6:103-110.

19. Getz GS, Reardon CA: Apoprotein E as a lipid transport and signaling protein in the blood, liver, and artery wall. Journal of Lipid Research 2009, 50:S156-S161.

20. Linton M, Atkinson J, Fazio S: Prevention of Atherosclerosis in Apolipoprotein EDeficient Mice by Bone Marrow Transplantation Science. 1995, 267:1034-1037.

21. Adams LA, Angulo P, Lindor KD: Nonalcoholic fatty liver disease. CMAJ 2005, 172(7):899-905.

22. Visser ME, Akdim F, Tribble DL, Nederveen AJ, Kwoh TJ, Kastelein JJP, et al: Effect of Apolipoprotein-B Synthesis Inhibition on Liver Triglyceride Content in Patients with Familial Hypercholesterolemia. Journal of lipid research 2010, 51(5):1057-1062.

23. Letteron P, Sutton A, Abdellah M, Fromenty B, Pessayre D: Inhibition of microsomal triglyceride transfer protein: another mechanism of druginduced steatosis in mice. Hepatology 2003, 38:133-140.

24. Hartmann G: Stahelin HB [Hyperlipidemia and atherosclerosis in Switzerland. Results from the "Basler studie" (author's transl)]. Ther Umsch 1980, 37:980-984.

25. Nakayama S, Sakashita M, Tonooka M, Gotoh H, Yasuhara H, Sakamoto K: Experimental hyperlipidemia and atherosclerosis induced by cholesterol diet in SPF Japanese white rabbits. Jpn J Pharmacol 1983, 33:279-289.

26. Rogers WR, Carey KD, McMahan CA, Montiel MM, Mott GE, Wigodsky HS, et al: Cigarette smoking, dietary hyperlipidemia, and experimental atherosclerosis in the baboon. Exp Mol Pathol 1988, 48:135-151.

27. Kuo PT: Hyperlipidemia in atherosclerosis. Dietary and drug treatment. Med Clin North Am 1970, 54:657-669.

28. Chang PY, Lu SC, Lee CM, Chen YJ, Dugan TA, Huang WH, et al: Homocysteine inhibits arterial endothelial cell growth through transcriptional downregulation of fibroblast growth factor-2 involving $G$ protein and DNA methylation. Circ Res 2008, 102:933-941.

29. Wang GP, Woo CWH, Sung FL, Siow YL OK: Increased monocyte adhesion to aortic endothelium in rats with hyperhomocysteinemia - Role of chemokine and adhesion molecules. Arterioscler Thromb Vasc Biol 2002, 22:1777-1783.

30. Scalia R, Gooszen ME, Jones SP, Hoffmeyer M, Rimmer DM 3rd, Trocha SD, et al: Simvastatin exerts both anti-inflammatory and cardioprotective effects in apolipoprotein E-deficient mice. Circulation 2001, 103:2598-2603.

31. Folch J, Lees M, Sloane Stanley GH: A simple method for the isolation and purification of total lipides from animal tissues. J Biol Chem 1957, 226:497-509.

32. Jiang MH, Fei J, Lan MS, Lu ZP, Liu M, Fan WW, et al: Hypermethylation of hepatic Gck promoter in ageing rats contributes to diabetogenic potential. Diabetologia 2008, 51:1525-1533.

\section{doi:10.1186/1476-511X-11-86}

Cite this article as: Chang et al:: The effects of berberine on hyperhomocysteinemia and hyperlipidemia in rats fed with a long-term high-fat diet. Lipids in Health and Disease 2012 11:86. 